\title{
Low Power Ultra-Wide Band Pulse Generator based on a Duty-Cycled 2-ASK Emitter
}

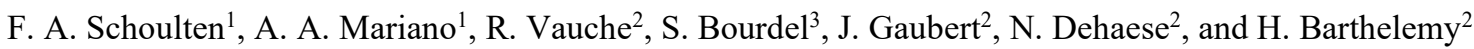 \\ ${ }^{1}$ Universidade Federal do Paraná, GICS-UFPR, Curitiba, Brazil \\ ${ }^{2}$ Aix-Marseille Univ, Univ Toulon, CNRS, IM2NP, France \\ ${ }^{3}$ Univ. Grenoble Alpes, Grenoble INP, RFIC-Lab, 38000 Grenoble, France
}

\begin{abstract}
This paper presents an ultra-wideband pulse generator based on a duty-cycled 2-ASK emitter able to generate OOK and BPSK modulated carriers. This emitter uses a differential architecture which has been designed in FDSOI $28 \mathrm{~nm}$ CMOS technology with a supply voltage of $1.0 \mathrm{~V}$. It presents a fast enough transient response to entirely cover the 3.1-10.6 GHz FCC band. Moreover, it can be quickly turnedon and turned-off which allow 3 ns pulses to be generated and also, low power impulse communications to be implemented. The emitter is built around a wideband Voltage Controlled Oscillator (VCO) which is able to generate at its outputs large oscillations from 1.0 GHz to $13.9 \mathrm{GHz}$. When the emitter is used as a $3 \mathrm{~ns}$ pulse generator centred on $7987 \mathrm{MHz}$, its energy consumption is $152.7 \mathrm{pJ} /$ pulse, and the static power consumption is $3.83 \mu \mathrm{W}$. This leads to a mean power consumption of $5.36 \mu \mathrm{W}$ (resp. $19.1 \mu \mathrm{W}$ and $156 \mu \mathrm{W}$ ) for a pulse repetition frequency of $10 \mathrm{kHz}$ (resp. $100 \mathrm{kHz}$ and 1 $\mathrm{MHz}$ ), which highlights the interest of impulse modulations for low power radio-frequency communications.
\end{abstract}

Keywords-BPSK; IEEE standards; Impulse Radio; Low Power; OOK; Power Gating; UWB; VCO.

\section{INTRODUCTION}

The eminent rise of the Internet of Things (IoT) along with the growing number of devices connected to Wireless Personal Area Network (WPAN) and Wireless Body Area Network (WBAN) will require wireless systems that must offer high-data quality transfer within short and medium range [1]. A promising technology that is well suited for WPAN and WBAN applications is the Ultra-wide Band Impulse Radio (IR-UWB), which is a communication technique based on short duration pulses transmission. The UWB communications have attracted interest of researchers since the Federal Communication Commission (FCC) has authorized the use of impulse modulations inside the UWB frequency band between $3.1 \mathrm{GHz}$ and $10.6 \mathrm{GHz}$ in 2002 [2$3]$. The duration of the associated pulses is typically the order of nanosecond and its energy can be spread on a frequency band of the order of $\mathrm{GHz}$.

The IR-UWB communications lead to a high energy efficiency on the transmitter side thanks to their duty-cycled nature. Indeed, the transmitter chain must be operational only during the pulses emission, which make it suitable for low power applications. Thus, the power consumption of UWB transmitters is proportional to data rate. Moreover, it is possible to produce short time domain pulses with simple and low power architecture. For this reason, the IR-UWB emitters can intrinsically present a low-power consumption which is reinforced by the low authorized power spectral densities that are orders of magnitude lower than mobile communications signals [4], in addition to high bit rate due to the use of short duration pulses.

Among the IR-UWB pulse generator reported architectures, one uses voltage-controlled oscillators (VCO) combined with a shaper circuit [5], meanwhile, other uses baseband pulses combiner, using delay cells [6]. Both works target an IR-UWB pulse generator whose generated pulse shape fits in spectral masks defined by the IEEE 802.15.4 standard, that defines 15 channels divided in 3 groups and whose bandwidth is between $499 \mathrm{MHz}$ and $1355 \mathrm{MHz}$ (Fig. 1). To be compliant with this standard, an UWB device must cover at least 1 channel from these 3 groups, where the mandatories channels are 0,3 and 9 .

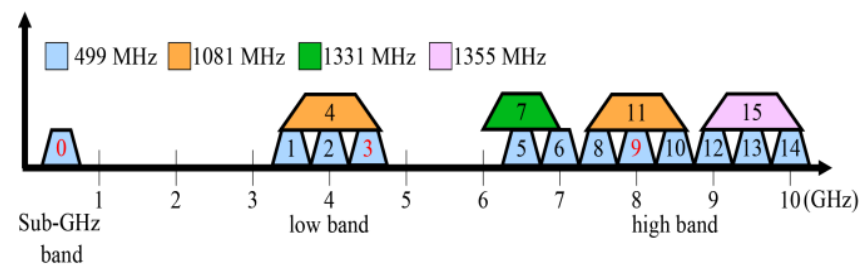

Fig. 1. IEEE 802.15.4a standard channels frequency plan.

In this paper, a 2-ASK emitter with a sufficient range to cover the FCC band (3.1-10.6 GHz) and which can be quickly turned-on and turned-off to be used as a pulse generator is reported. It consists in a VCO followed by a buffer stage and an antenna buffer. The VCO can be switched on and off through a logic signal and its quick response to start or stop dispenses the use of a mixing stage. Moreover, this VCO has a differential output voltage whose phase can be quickly commuted, which enables bipolar modulations such as BPSK. Therefore, it can be used for IRUWB communications as well as for the traditional 2-ASK communications based on a modulated carrier. Finally, although communications based on carrier modulation require an oscillator operating all the time, an interesting feature of this circuit is that it can be powered down between two consecutives emitted pulses and then consumes only during a pulse transmission.

This paper is organized as follows. Section II discusses IR-UWB pulse generation and presents the pulse generator architecture. Section III focuses on the differential VCO architecture designed with a FD-SOI $28 \mathrm{~nm}$ design kit. In section IV, simulation results demonstrate that the proposed emitter covers all the 3.1-10.6 GHz band and can be used for IR-UWB pulse generation.

\section{IR-UWB PULSE GENERATION}

\section{A. Pulse generator architecture}

For an IR-UWB transmission, the data can be transmitted using different values of the emitted pulses parameters like amplitude, position, or polarity. In the case of a pulse amplitude modulation (PAM), the signal emitted by the UWB antenna can be expressed as a combination of single pulses, such that: 


$$
s(t)=\sum_{k=0}^{\infty} d_{k} \cdot p\left(t-\frac{k}{D_{s}}\right)
$$

where $d_{k}$ is the $k^{\text {th }}$ emitted symbol taken from a M-ary alphabet $\mathrm{D}, D_{s}$ is the symbol rate, and $p(t)$ is the generated pulse waveform. For an OOK modulation, the alphabet $D$ is:

- $D:\left\{d_{k}\right\}=\{0,1\}$

and for an BPSK modulation, the alphabet $D$ is:

$$
\text { - } D:\left\{d_{k}\right\}=\{-1,1\} \text {. }
$$

The signal from (1) can also be generated by the UWBIR emitter based on gated oscillator as proposed here, whose architecture is displayed in Fig. 2. The main parameters of the pulse generation by an oscillator are the oscillation duration $\tau_{p}$ and the oscillation frequency $f_{m}$, that respectively determines the generated pulse bandwidth and its central frequency. To charge a $50 \Omega$ UWB antenna, a buffer stage is required and consists here of push-pull circuit and differential buffers. The buffer stage and antenna, placed right after the differential $\mathrm{VCO}$, are not detailed in this paper.

In order to achieve a pulse with a central frequency inside the UWB FCC band, the emitter proposed here is built around a differential VCO having a frequency control, and start-up and stop command. The pulse duration is controlled here by the time that the oscillator is operational (signal "Enable" with high logic level) and the VCO frequency can be largely tuned using "Voltage Control". To enable BPSK modulations, the output phases can be exchanged using the logic signal "Phase". Finally, the emitter can be turned-on or turned-off through the logic signal "ON/OFF".

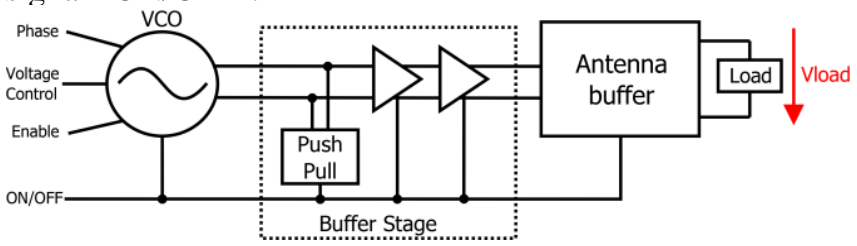

Fig. 2. Proposed IR-UWB emitter based on gated oscillator architecture.

\section{B. Pulse waveform generated by gated oscillators}

For a gated oscillator generating sinusoidal waveform signals, the pulse shape $p(t)$ can be written such that:

$$
\begin{gathered}
p(t)=A \cdot \sin \left(2 \pi f_{m} t\right) \cdot \Pi(t) \\
\Pi(t)=\left\{\begin{array}{lll}
1 & \text { if } & -\tau_{p} / 2 \leq t \leq \tau_{p} / 2 \\
0 & \text { if } & \text { otherwise }
\end{array}\right.
\end{gathered}
$$

where $A$ is the maximum pulse magnitude and $\Pi(\mathrm{t})$, the rectangular function having a width of $\tau_{p}$. Notice that the pulse shape is here a sinusoidal waveform with a rectangular windowing, with a duration controlled. Shifting to frequency domain, the mono-lateral Fourier transform of the emitted pulse can be expressed as:

$$
P^{+}(f)=\frac{A \tau_{p}}{\sqrt{2}} \operatorname{sinc}\left(\pi\left(f-f_{m}\right) \tau_{p}\right) \exp (-j \varphi)
$$

where $\varphi$ is the sinewave phase. For a BPSK impulse modulation, the power spectral density is proportional to the squared magnitude $P^{+}(f)$.
The duration $\tau_{p}$ of $p(t)$ can be expressed as a function of the oscillation frequency $f_{m}$ as follows:

$$
\tau_{p}=\frac{N}{2 f_{M}}
$$

where $N$ is the integer number of sinusoidal half-periods of the generated pulse. However, the minimal number of halfperiods of the sinewave, in relation of the bandwidth $B W_{-X d B}$, can be written:

$$
N_{\min }=\alpha \frac{f_{M}}{B W_{-X d B}}
$$

where $\alpha$ is a coefficient that has a value of $1.8,3$ and 10.8 when the attenuation $X d B$ is $3 \mathrm{~dB}, 10 \mathrm{~dB}$ and $18 \mathrm{~dB}$ respectively. From (5) and (6), the pulse minimum width can be expressed as:

$$
\tau_{p-\min }=\frac{\alpha}{2 B W_{-X d B}}
$$

Finally, it can be noted that an attenuation of $10 \mathrm{~dB}$ is enough to be compliant with FCC requirements. However, an attenuation of $18 \mathrm{~dB}$ is for example necessary to be compliant with the IEEE 802.15.4 standard.

\section{PROPOSED VCO DESIGN}

The gated voltage-controlled oscillator is the main circuit of the 2-ASK emitter which allows this last to be used as an IR-UWB pulse generator. It is based on a ring oscillator architecture whose principle is to propagate a signal on this close loop chain. Thus, it consists here of an odd number of cascaded delay cells, connected in a close loop chain, as indicated in the Figs. 3 and 4. This architecture is interesting given that it does not require inductor neither capacitor and it is able to provide high frequency oscillations, and multiphase outputs to the detriment of the phase noise. The instability condition, required for oscillations start, comes from the number of delay cells, where the minimum necessary is two for differential architecture, and three for non-differential architecture. Indeed, a ring with one delay cell is not feasible because the transistor will be self-biased, presenting only a DC component current. Also, the delay time of cells defines the oscillation frequency. The proposed VCO design includes buffer cells, in order to make the oscillation frequency independent of the VCO load.

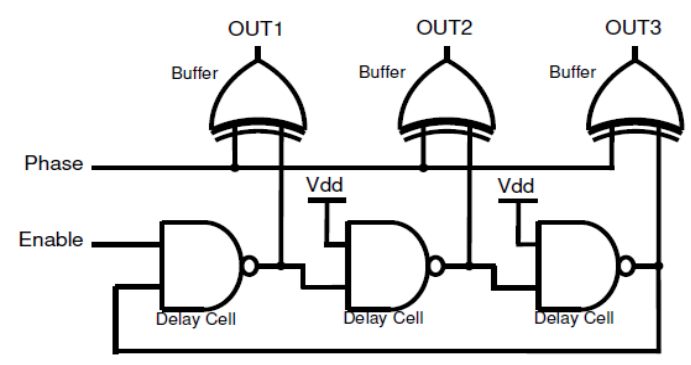

Fig. 3. Proposed VCO topology.

The delay cell and buffer cell schematic are presented in Figs. 5 and 6. "Vctrl" is a voltage which control the oscillation frequency. The signals "ON" and "OFF" control the pulse duration, while the signal "PDB" turns-on or turnsoff the IR-UWB pulse generator. Meanwhile, the signals "INV" and "INVV" control the VCO output signal phase. The truth table of the delay cell and buffer cell are displayed in 
Table I. The push-pull, placed between the gated VCO and the buffer stage, has been added to amplify the VCO output current, thus providing a better signal dynamic and improving the driving capability especially for high frequency.

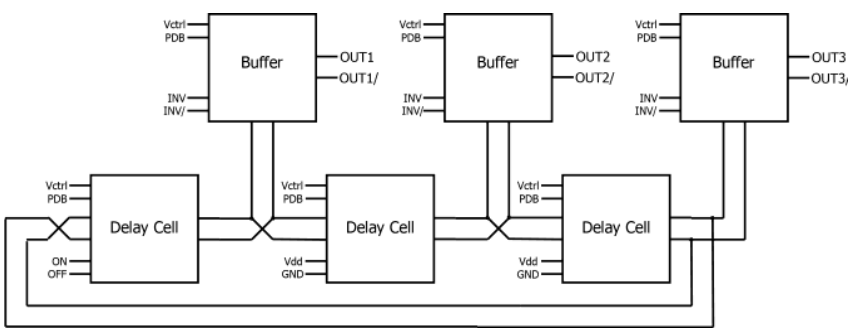

Fig. 4. Proposed gated differential VCO architecture.

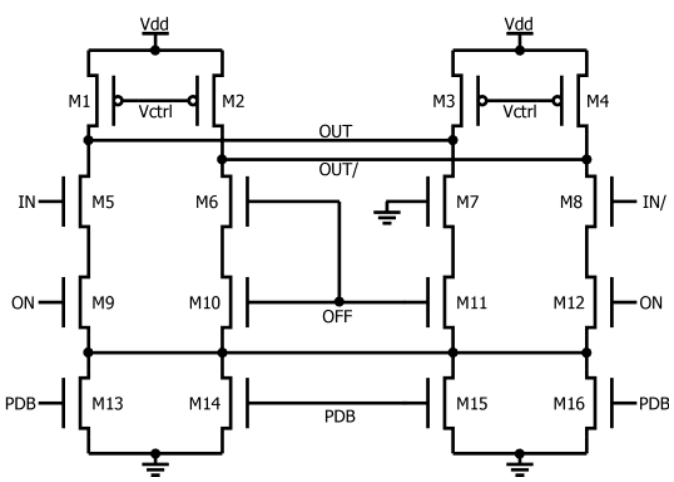

Fig. 5. VCO delay cell schematics.

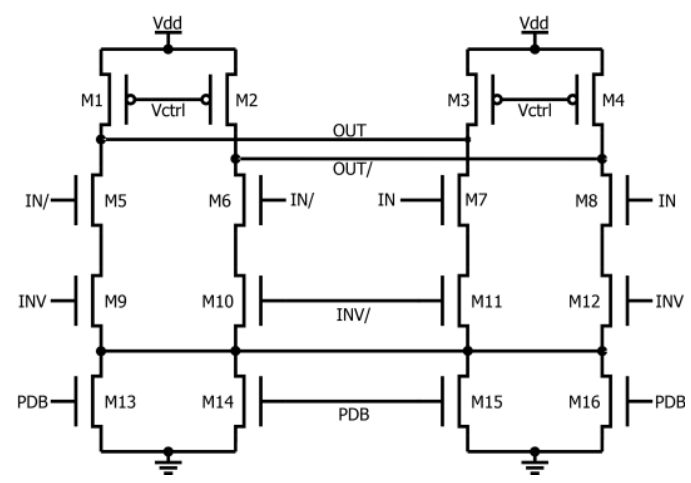

Fig. 6. VCO buffer cell schematics.

TABLE I. VCO CELLS TRUTH TABLES

\begin{tabular}{|c|c|c|c|c|c|}
\hline \multicolumn{3}{|c|}{ Delay Cell } & \multicolumn{3}{c|}{ Buffer Cell } \\
\hline IN & ON & OUT & IN & INV & OUT \\
\hline 0 & 0 & 0 & 0 & 0 & 0 \\
\hline 0 & 1 & 0 & 0 & 1 & 1 \\
\hline 1 & 0 & 0 & 1 & 0 & 1 \\
\hline 1 & 1 & 1 & 1 & 1 & 0 \\
\hline
\end{tabular}

\section{Simulation Results}

This section presents simulation results of the 2-ASK emitter when it is used as a pulse generator. It has been designed using the FD-SOI $28 \mathrm{~nm}$ CMOS design kit from STMicroeletronics, with a supply voltage of $1.0 \mathrm{~V}$. In the design process, the possible parasitic capacitances at each node have been considered. The values of the parasitic capacitances were extracted from a parasitic extraction of another previous design.

Fig. 7 shows the VCO frequency and its always-on power consumption as a function of the oscillation frequency control voltage ("Vctrl"),, in addition to the always-on power consumption of the IR-UWB emitter. From Fig. 7, it can be noticed that the proposed design is able to cover all the 3.1-10.6 GHz, where the maximum frequency reached is $13.2 \mathrm{GHz}$. The average (resp. minimum and maximum) $\mathrm{VCO}$ gain is about $-18.1 \mathrm{GHz} / \mathrm{V}$ (resp. - $20.8 \mathrm{GHz} / \mathrm{V}$ and $-10.6 \mathrm{GHz} / \mathrm{V}$ ). When "Enable" and "PDB" have a high logic level, the always-on power consumption of the proposed IR-UWB emitter and the gated oscillator have a value of $45.7 \mathrm{~mW}$ and $11.1 \mathrm{~mW}$ respectively, for a VCO frequency of $7987 \mathrm{MHz}$. When "Enable" and "PDB" has a low logic level, the pulse generator is turned-off and has a total power consumption of $3.83 \mu \mathrm{W}$. Thanks to the oscillation frequency range, the proposed pulse generator is able to address the central frequencies of all the IEEE 802.15.4 channels.

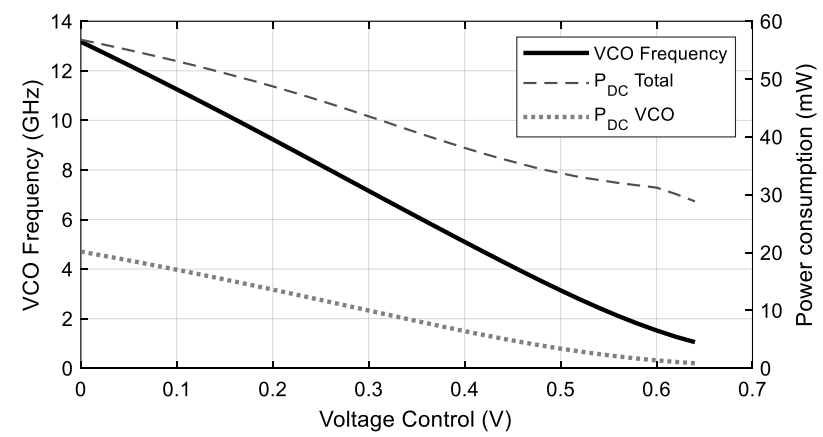

Fig. 7. VCO frequency and IR-UWB emiter always-on power consumtpion in terms of VCO voltage control.

In Fig. 8 and Fig. 9, pulses obtained through transient simulations and targeting a central frequency similar to the ones of the mandatory channels 3 (4482 MHz) and 9 (7987 $\mathrm{MHz}$ ) of IEEE 802.15.4 standard are shown. They have a bandwidth $B W_{X=-10 d B}(\alpha=3)$ equals to $500 \mathrm{MHz}$ and also can be used outside the context of IEEE standards as allowed by FCC requirements since pulses spectrum side lobes present a power spectrum density lower than $10 \mathrm{dBc}$. However, this is not enough to be fully compliant with IEEE standards. For this reason, an additional pulse shaper circuit should be added to the proposed pulse generator to synthesize a pulse with a particular envelop which fulfils in the same time IEEE standards requirements and the FCC ones.

To obtain a $10 \mathrm{~dB}$ bandwidth of $500 \mathrm{MHz}, \alpha$ has been set to 3 which leads using (7) to $\tau_{p}=3$ ns. Thus, the "Enable" input must have a high logic level for $3 \mathrm{~ns}$, given that the VCO has a fast response time. Regarding the simulations, signal "ON" from Fig. 4 (resp. signal "PDB" from Fig. 4) have high logic level for $3 \mathrm{~ns}$ (resp. $3.6 \mathrm{~ns}$ ). It can be noted that the PDB high level duration must be higher than the ON high level duration in order to let biasing currents and voltages to be initialized. Finally, when DATA $=1$, the signal "INV" has low logic level, otherwise, the signal "INV" has high logic level which allows DATA to change the generated pulse phase to $180^{\circ}$ (BPSK modulation). As shown in Fig. 8 and Fig. 9, it can be noticed, for the same central frequency, that the two pulses with different value of DATA are exactly in phase opposition and therefore have the same frequency 
response. Given that the IR-UWB signals presents a gated nature, the average power consumption can be expressed in terms of the pulse repetition frequency (PRF), such that:

$$
P_{c}(P R F)=E_{A C} \cdot P R F+P_{D C}
$$

where $E_{A C}$ is the energy consumed per generated pulse, and $P_{D C}$ the static power that is consumed when no pulse is generated. It can be noted that this last is here equal to the leakage power consumption since the pulse generator circuit can be fully turned-off. Both parameters $E_{A C}$ and $P_{D C}$ can be extracted from at least two measured average power consumption at different values of PRF. Moreover, (8) highlights the particularity of impulse communications which is that the power consumption increases with the data rate. For the proposed pulse generator, when 3 ns pulses centred at $7987 \mathrm{MHz}$ are generated, $P_{c}$ is equal to $1.53 \mathrm{~mW}$ (resp. $15.27 \mathrm{~mW}$ ) for a PRF of $10 \mathrm{MHz}$ (resp. $100 \mathrm{MHz}$ ), which leads to an a $E_{A C}$ of $152.7 \mathrm{pJ} /$ pulse and a $P_{D C}$ of 3.33 $\mu \mathrm{W} . P_{D C}$ is also very close to the stand-by power consumption $(3.83 \mu \mathrm{W})$ of the pulse generator, the small difference coming from the precision with which $P_{c}$ was obtained in simulation. Thus, the power consumption of the proposed pulse generator for this type of pulses is $5.36 \mu \mathrm{W}$ (resp. $19.1 \mu \mathrm{W}$ and $156 \mu \mathrm{W}$ ) for a pulse repletion frequency of $10 \mathrm{kHz}$ (resp. $100 \mathrm{kHz}$ and $1 \mathrm{MHz}$ ). Finally, a comparison with the state of the art is done in table II. It can be noted that the proposed pulse generator is the only one which is able to cover all the FCC band (3.1-10.6 GHz) in addition to have the greatest output voltage amplitude level.
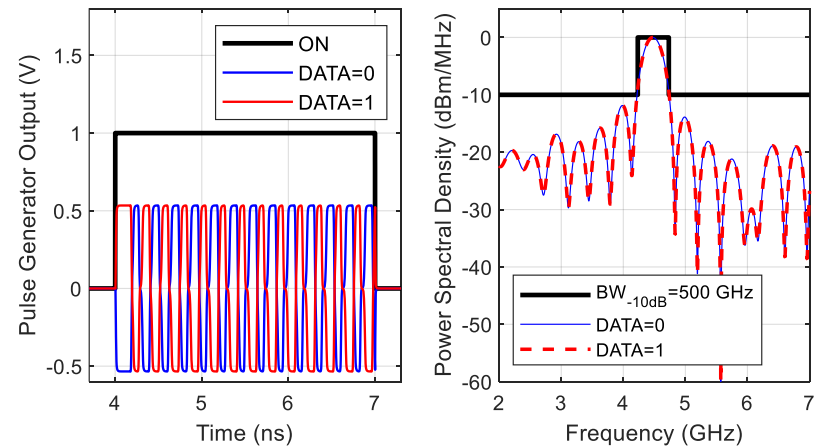

Fig. 8. IR-UWB pulse generator simulated response for a $\mathrm{BW}_{-10 \mathrm{~dB}}$ of 500 $\mathrm{MHz}\left(\tau_{\mathrm{p}-\min }=3 \mathrm{~ns}\right)$ centered on $4482 \mathrm{MHz}$ (channel 3).
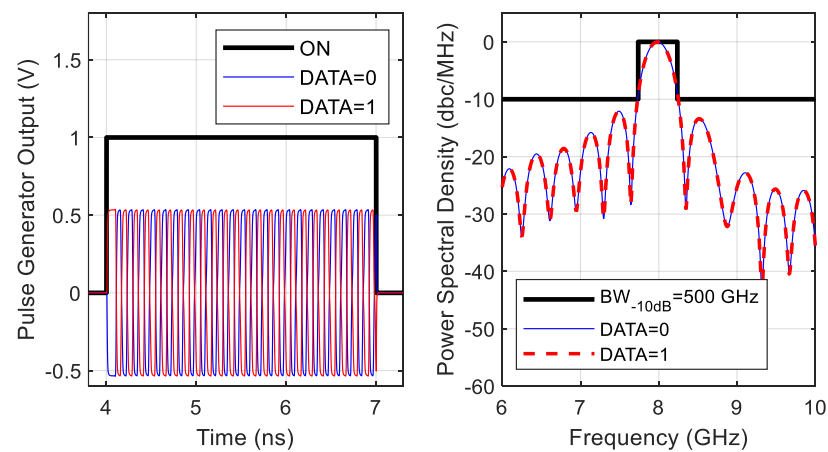

Fig. 9. IR-UWB pulse generator simulated response for a BW-10dB of 500 $\mathrm{MHz}(\tau \mathrm{p}-\mathrm{min}=3 \mathrm{~ns})$ centered on $7987 \mathrm{MHz}$ (channel 9).

\section{CONCLUSION}

In this paper, a low power IR-UWB pulse generator based on a duty-cycled 2-ASK emitter has been presented. It is composed of a gated and differential voltage-controlled oscillator and a buffering stage. This circuit has been designed in FD-SOI $28 \mathrm{~nm}$ CMOS technology from STMicroelectronics. The simulations results show that the proposed gated VCO is fully capable to cover the 3.1-10,6 $\mathrm{GHz}$ band, regulated by the FCC, and thus to potentially address every channel of the IEEE 802.15.4 standard. Nevertheless, to be fully compliant with the IEEE standard spectrum requirements, an additional pulse shaper circuit is required and should be designed. In terms of power consumption, the VCO achieves an always-on power consumption of $11.1 \mathrm{~mW}$ whereas the full pulse generator has an always-on power consumption of $45.7 \mathrm{~mW}$. Finally, the pulse generator has an energy consumed per pulse of 152.7 pJ when it generates 3 ns pulses centred on 7987 $\mathrm{MHz}$ and a static power consumption of $3.83 \mu \mathrm{W}$ (leakage consumption). Therefore, the circuit total power consumption can be reduced until this limit by reducing the data rate.

TABLE II. STATE OF THE ART COMPARISON

\begin{tabular}{|c|c|c|c|c|c|}
\hline & $\begin{array}{c}\text { This } \\
\text { Work }^{\mathrm{a}}\end{array}$ & [5] & [6] & [7] & [8] \\
\hline Techno. & FD-SOI & $65 \mathrm{~nm}$ & $130 \mathrm{~nm}$ & $65 \mathrm{~nm}$ & $90 \mathrm{~nm}$ \\
\hline $\begin{array}{c}\text { Power Supply } \\
\text { (V) }\end{array}$ & 1 & 1.2 & 1.2 & 1.2 & 0.9 \\
\hline $\mathrm{Fc}(\mathrm{GHz})$ & $3-12$ & $3-5$ & $3-5$ & $6.5-8$ & $3.1-5$ \\
\hline $\mathrm{BW}(\mathrm{X}=3 \mathrm{~dB})$ & $0.5-1.3$ & $0.5-1.3$ & $0.5-1.3$ & 0.5 & $0.5-1.4$ \\
\hline $\begin{array}{c}\text { Amplitude } \\
\text { (V) }\end{array}$ & 0.53 & 0.4 & 0.45 & 0.45 & $\begin{array}{c}0.05- \\
0.06 \\
\end{array}$ \\
\hline $\begin{array}{c}\text { Eac } \\
\text { (pJ/pulse) }\end{array}$ & 152.7 & 1.9 & 146 & 2.1 & $8-12$ \\
\hline $\begin{array}{c}\text { Static power } \\
(\mu \mathrm{W})\end{array}$ & 3.83 & NA & 100 & 0.48 & 170 \\
\hline Architecture & $\begin{array}{c}\text { LO } \\
\text { based }\end{array}$ & $\begin{array}{c}\text { LO } \\
\text { based }\end{array}$ & $\begin{array}{c}\text { Edge } \\
\text { Combiner }\end{array}$ & $\begin{array}{c}\text { Edge } \\
\text { Combiner }\end{array}$ & $\begin{array}{c}\text { LO } \\
\text { based }\end{array}$ \\
\hline
\end{tabular}

\section{REFERENCES}

[1] J. Wang and Q. Wang. Body Area Communications: Channel Modeling, Communication Systems, and EMC. IEEE Press, Piscataway, NJ, USA, $1^{\text {st }}$ edition, 2013.

[2] Federal Communications Commission. FCC rules and regulations. Part 15, July 2002.

[3] Matti Hamalainen Ian Oppermann and Jari Iinattin. UWB Theory and Applications. John Wiley Sons, University of Oula, Finland, 2004. ISBN 0-470-86917-8.

[4] A. Goavec, M. Zarudniev, R. Vauché, F. Hameau, J. Gaubert, and E. Mercier. An efficient method of power spectral density estimation for on-chip IR-UWB transmitter self-calibration. IEEE Transactions on Circuits and Systems I: Regular Papers, 64(3):686-695, March 2017.

[5] F. Artemio-Schoulten et al., "Pulse Design Method for Complexity Reduction of IEEE IR-UWB Pulse Synthesizers," 2020 27th IEEE International Conference on Electronics, Circuits and Systems (ICECS), Glasgow, UK, 2020, pp. 1-4..

[6] [5] R. Vauche et al., "A $100 \mathrm{MHz}$ PRF IR-UWB CMOS Transceiver With Pulse Shaping Capabilities and Peak Voltage Detector," in IEEE Transactions on Circuits and Systems I: Regular Papers, vol. 64, no. 6, pp. 1612-1625, June 2017.

[7] T. Haapala and K. Halonen. A fully integrated digitally programmable pulse shaping $6.0-8.5 \mathrm{GHz}$ UWB IR transmitter frontend for energy harvesting applications. In 2018 IEEE International Symposium on Circuits and Systems (ISCAS), pages 1-5, May 2018.

[8] Y. Park and D. D. Wentzloff. An all-digital $12 \mathrm{pJ} /$ pulse IR-UWB transmitter synthesized from a standard cell library. IEEE Journal of Solid-State Circuits, 46 (5):1147-1157, May 2011. 